\title{
Neutrophils activate tumoral CORTACTIN to enhance progression of orohypopharynx carcinoma
}

\author{
Claudia A. Dumitru ${ }^{1}$, Agnes Bankfalvi ${ }^{2}$, Xiang Gu ${ }^{1}$, Wilfried E. Eberhardt ${ }^{3}$, Reinhard Zeidler ${ }^{4}$, Stephan Lang ${ }^{1}$ \\ and Sven Brandau ${ }^{1 *}$
}

${ }^{1}$ Department of Otorhinolaryngology, University Hospital Essen, University of Duisburg-Essen, Essen, Germany

${ }^{2}$ Department of Pathology/Neuropathology, University Hospital Essen, University of Duisburg-Essen, Essen, Germany

${ }^{3}$ Department of Medical Oncology, West German Cancer Centre, University Hospital Essen, University of Duisburg-Essen, Essen, Germany

${ }^{4}$ Department of Otorhinolaryngology, Ludwig Maximilians University, Munich, Germany

\section{Edited by:}

Jean-Pierre Abastado, Agency for Science, Technology and Research, Singapore

\section{Reviewed by:}

Lai Guan Ng, Agency for Science, Technology and Research, Singapore Kar Wai Tan, Singapore Immunology Network, Singapore

\section{*Correspondence:}

Sven Brandau, Department of Otorhinolaryngology, University of Duisburg-Essen, Hufelandstraße 55, 45122 Essen, Germany.

e-mail: sven.brandau@uk-essen.de
CORTACTIN is an actin-binding protein critically involved in cellular migration and invasion. Here, we investigated the role of CORTACTIN in the pathophysiology of orohypopharynx carcinoma - one of the major subtypes of head and neck cancer. To this end, we analyzed CORTACTIN expression in tumor tissues from 89 orohypopharynx carcinoma patients in relation to clinical parameters. We found that high tumoral CORTACTIN expression associated with poor survival, higher T-stage, and higher lymph node metastasis ( $\mathrm{N}$-stage) in these patients. Next, we combined the prognostic values of tumoral and stromal cell biological parameters in our patient cohort. We determined the potential interaction of tumoral CORTACTIN with tumor-infiltrating neutrophils, which have been previously linked to poor clinical outcome in orohypopharynx carcinoma patients with advanced disease. Interestingly, we found that patients with both high tumoral CORTACTIN expression and high neutrophilic infiltration had significantly worse clinical outcome than all other patients in our cohort. These findings suggest that tumoral CORTACTIN and tumor-infiltrating neutrophils might be functionally linked during progression of orohypopharynx carcinoma. In vitro, we showed that neutrophils released soluble factors which phosphorylated CORTACTIN in the tumor cells and promoted their migration. Furthermore, we demonstrated that strong CORTACTIN phosphorylation significantly correlated with strong neutrophilic infiltration in tumor tissues from orohypopharynx carcinoma patients. Taken together, our findings unravel a novel mechanism of tumor-stroma interaction, which might be relevant for a more accurate prognosis and improved therapeutic strategies in this tumor entity.

Keywords: cancer-related inflammation, tumor microenvironment, head and neck cancer, clinical outcome, tumor migration

\section{INTRODUCTION}

Head and neck cancer (HNC) is the eighth most common type of cancer worldwide. Despite multiple and aggressive therapeutic interventions, there has been no fundamental improvement in the 5 -year survival rates of the patients over the past decades (Jemal et al., 2007; Choong and Vokes, 2008). Therefore, there is an urgent need to: (i) identify clinicopathological parameters (biomarkers) for accurate diagnosis, prognosis, and therapeutic prediction in these patients and to (ii) understand the biology and molecular mechanisms behind the respective biomarkers.

A number of studies investigated the role of cortical actinbinding protein (CORTACTIN) as prognostic marker in different types of cancer. CORTACTIN is a cytoplasmic protein which promotes the rearrangement of the actin cytoskeleton. Consequently, CORTACTIN is critically involved in regulation of cellular motility/migration, invasion, or endocytosis/vesicular trafficking (reviewed in Ammer and Weed, 2008). To exert its functions, CORTACTIN needs to be activated by tyrosine or serine/threonine phosphorylation. In particular, tyrosine phosphorylation of CORTACTIN at residues Tyr 421, Tyr 466, and Tyr 482 has often been shown to occur in response to various stimuli (Ammer and Weed,
2008). Consistent with its role in cellular migration and invasion, high CORTACTIN expression and/or activation has been found to associate with increased metastasis and poor clinical outcome in several types of cancer, such as renal carcinoma (Wang et al., 2009), gastric cancer (Li et al., 2008), colorectal adenocarcinoma (Cai et al., 2010), or melanoma (Xu et al., 2010). In HNC, amplification of CORTACTIN gene has been linked to poor clinical outcome mainly in patients with laryngeal carcinoma (Gibcus et al., 2008; Rodrigo et al., 2009).

Solid tumors display an inflammatory microenvironment, characterized by large numbers of tumor-associated immune cells, which represent a biologically important component of the tumorstroma (Coussens and Werb, 2002; Lin and Karin, 2007). Within the tumor microenvironment, cancer cells can "reprogram" the stromal immune cells of the host to acquire tumor-promoting activities. Although less characterized than tumor-associated macrophages (TAMs) or tumor-infiltrating lymphocytes (TILs), tumor-infiltrating neutrophils are emerging as important players in the pathophysiology of cancer. Within the tumor tissue, neutrophils can potentially modulate multiple cellular processes which may ultimately lead to tumor progression. In particular, 
neutrophils were shown to promote angiogenesis (Nozawa et al., 2006; Jablonska et al., 2010; Bekes et al., 2011; Kuang et al., 2011). However, neutrophils can directly modulate the biology and functions of tumor cells by promoting their migration, invasion, or proliferation (reviewed in Gregory and Houghton, 2011; Dumitru et al., 2012b). Thus, it is not surprising that very recent studies reported an association of high numbers of tumor-infiltrating neutrophils with advanced disease and poor clinical outcome in patients with different types of cancer, such as renal cancer (Donskov and von der Maase, 2006; Jensen et al., 2009), hepatocellular cancer (Kuang et al., 2011), non-small-cell lung carcinoma (NSCLC; Ilie et al., 2012), or melanoma (Jensen et al., 2012). Recently, we demonstrated that high neutrophilic infiltration of the tumor tissue associated with high tumor $(\mathrm{T})$ stage and poor survival in head and neck (orohypopharynx) cancer patients with advanced disease (Trellakis et al., 2011). Furthermore, our in vitro studies indicated a direct interaction between neutrophils and HNC cells by showing that neutrophils were "primed" by the tumor cells to release pro-inflammatory factors which promoted tumoral migration in a feed-back manner (Dumitru et al., 2011, 2012a).

In the present study we characterized the tumor-neutrophil interactions in orohypopharyngeal cancer with particular focus on the role of tumoral CORTACTIN in this process. We demonstrate that high tumoral CORTACTIN levels together with high neutrophilic infiltration predict poor clinical outcome in univariate and multivariate analysis. These findings suggest that tumoral CORTACTIN and tumor-infiltrating neutrophils "cooperate" in the progression of orohypopharynx carcinoma. Further in vitro and in situ studies confirm the clinical findings and demonstrate that neutrophils activate (phosphorylate) CORTACTIN in the tumor cells to promote their migration.

\section{MATERIALS AND METHODS STUDY SUBJECTS}

Neutrophils were isolated from the peripheral blood of healthy volunteers. For immunohistochemical studies on frozen sections, malignant tissues were collected from 37 patients with orohypopharyngeal cancer, treated at the Department of Otorhinolaryngology (University of Duisburg-Essen) between 2010 and 2012. Healthy epithelial tissues $(n=12)$ were collected from contralateral buccal mucosa of the same patients. For immunohistochemical studies on tissue microarrays (TMAs) and correlation with clinical parameters, tissue samples were collected from 89 patients with head and neck squamous cell carcinoma of the oropharynx and hypopharynx. The patients were treated at the Department of Otorhinolaryngology (University of Duisburg-Essen) between 1995 and 2000 and clinical follow-up was retrieved. The characteristics of these patients are shown in Table 1. All studies were approved by the ethics committee of the University Hospital Essen (nr. 09-4074, nr. 07-3500, and nr. 12-5192-BO).

\section{CELL LINES, CONDITIONED SUPERNATANTS, AND SIRNA TRANSFECTIONS}

The human hypopharyngeal carcinoma cell line FaDu was obtained from the American Type Culture Collection (ATCC,
Table 1 | Characteristics of the patients used for TMA analysis and association with clinical parameters (survival, $\mathrm{T}$-stage, and $\mathrm{N}$-stage).

\begin{tabular}{lcc}
\hline & Number & \% of total \\
\hline All patients & 89 & 100 \\
GENDER & & \\
Male & 71 & 79.8 \\
Female & 18 & 20.2 \\
TUMOR LOCALIZATION & & \\
Oropharynx & 65 & 73.0 \\
Hypopharynx & 24 & 27.0 \\
T-STAGE & & \\
T1 & 13 & 14.6 \\
T2 & 19 & 21.3 \\
T3 & 12 & 13.5 \\
T4 & 45 & 50.6 \\
N-STAGE & & \\
NO & 14 & 15.7 \\
N1 & 13 & 14.6 \\
N2a & 6 & 6.7 \\
N2b & 20 & 22.5 \\
N2C & 32 & 36.0 \\
N3 & 4 & 4.5 \\
DISTANT METASTASIS & & 6.7 \\
MO & 86 & 31.5 \\
M1 & 3 & 3.4 \\
HISTOLOGIC GRADE & & \\
Grade 1 & 65 & \\
Grade 2 & & \\
Grade 3 & & \\
\hline & & \\
\hline
\end{tabular}

Manassas, VA, USA). The cells were cultured and maintained in RPMI-1640 (Invitrogen, Karlsruhe, Germany) supplemented with $10 \%$ fetal calf serum (Biochrom, Berlin, Germany), $100 \mathrm{units} / \mathrm{mL}$ penicillin, and $100(\mathrm{~g} / \mathrm{mL}$ streptomycin (PAA-Laboratories GmbH, Coelbe, Germany). To obtain tumor-conditioned supernatants $(\mathrm{FaDu} \mathrm{SN})$, we incubated $2 \times 10^{6}$ cells $/ \mathrm{mL}$ for $24 \mathrm{~h}$ at $37^{\circ} \mathrm{C}$ in RPMI- 1640 supplemented as above. The resulting supernatant was centrifuged to remove cellular debris and stored at $-20^{\circ} \mathrm{C}$.

To knock-down CORTACTIN, we transfected FaDu cells with $25 \mathrm{nM}$ validated CORTACTIN siRNA or $25 \mathrm{nM}$ AllStars Negative Control (mock) siRNA (both from Qiagen, Hilden, Germany) using the $\mathrm{NEON}^{\mathrm{TM}}$ transfection system (Invitrogen, Karlsruhe, Germany).

\section{MIGRATION ASSAY}

Migration of FaDu cells was assessed with the ORIS ${ }^{\mathrm{TM}}$ cell migration system (Platypus Technologies, Madison, WI, USA) according to the manufacturer's instructions. The cells were allowed to migrate for $48 \mathrm{~h}$ and were then fixed/stained with a solution containing $2 \%$ formaldehyde, $30 \%$ ethanol, $60 \mathrm{mM} \mathrm{NaCl}$, and $0.7 \%$ crystal violet. Micrographs were taken at $25 \times$ magnification and quantification of "gap"-closure was performed with the ImageJ software. 


\section{ISOLATION AND CULTURE OF NEUTROPHILS}

Neutrophils (purity $>98 \%$ ) were isolated from the blood of healthy donors as previously described (Dumitru et al., 2011) and were cultured in RPMI-1640 supplemented as above.

\section{AMIDA SCREENING}

Tumors elicit an immune response, leading to the generation of antibodies specific for tumoral antigens. AMIDA technology (autoantibody-mediated identification of antigens) identifies proteins exclusively recognized by antibodies from cancer patients but not from healthy controls. Here, we used AMIDA to screen for tumor-associated antigens in four HNC patients versus four healthy donors. The screening was performed exactly as described previously (Rauch et al., 2004).

\section{ANTIBODIES AND INHIBITORS}

Polyclonal rabbit anti-human CORTACTIN and rabbit IgG antibodies were obtained from Santa Cruz Biotechnology (Santa Cruz, CA, USA). Rabbit anti-human phospho-CORTACTIN (Tyr421) antibodies were from Cell Signaling Technology (Danvers, MA, USA) or from Millipore (Temecula, CA, USA). Rabbit anti-human GAPDH was from Cell Signaling Technology. Mouse anti-human CD66b antibodies were obtained from Immunotech (Marseille Cedex 9, France). The secondary antibodies (DyLight 488-donkey anti-rabbit, HRP-goat anti-rabbit, HRP-donkey anti-goat, AP-goat anti-rabbit) were from Dianova (Hamburg, Germany) while Alexa 546-goat anti-mouse IgG was from Molecular Probes (Leiden, The Netherlands). Protease inhibitor cocktail sets I and III were from Merck (Darmstadt, Germany). The phosphatase inhibitor cocktail PhosStop was from Roche Diagnostics (Mannheim, Germany).

\section{IMMUNOHISTOCHEMISTRY AND IMMUNOFLUORESCENCE}

Frozen sections of tumor or healthy epithelial tissue were fixed with BD Cytofix/Cytoperm and incubated with primary antibodies overnight at $4^{\circ} \mathrm{C}$. Secondary (and tertiary reactions, for colorimetric immunohistochemistry) were performed for $30 \mathrm{~min}$ each at room temperature. Nuclei were counterstained using Hematoxylin - for immunohistochemistry (Thermo Scientific, Karlsruhe, Germany) or DRAQ5 - for immunofluorescence (eBioscience, San Diego, CA, USA). Samples were finally mounted in Kaisers glycerol gelatine (Merck, Darmstadt, Germany) or in Fluoprep (bioMerieux, Marcy l'Etoile, France) and analyzed with a Zeiss Axioskop 2 microscope (Zeiss, Jena, Germany).

For TMAs, immunohistochemical staining was performed with an automated staining device (Dako Autostainer; DakoCytomation, Hamburg, Germany) using rabbit anti-human CORTACTIN antibodies. Secondary and tertiary immunoreactions were performed with commercially available anti-rabbit IgG detection kit (En-Vision; DakoCytomation, Hamburg, Germany). Analysis was performed with a Zeiss Axioscope 2 microscope. Staining and analysis of TMAs with CD66b antibodies has been described previously (Trellakis et al., 2011).

\section{FLOW CYTOMETRY}

To assess the levels of CORTACTIN after siRNA transfection, FaDu cells were fixed with BD Cytofix/Cytoperm and incubated with rabbit anti-CORTACTIN or isotype control antibodies for $1 \mathrm{~h}$ at room temperature. Secondary reactions were performed for 20 min at room temperature using DyLight488-conjugated antirabbit antibodies. Samples were analyzed by flow cytometry with a BD FACSCanto II flow cytometer (BD, Heidelberg, Germany).

\section{SDS-PAGE AND WESTERN BLOT ANALYSIS}

FaDu cells $\left(10^{6}\right.$ cells/sample) were lysed with a buffer containing $25 \mathrm{mM}$ HEPES ( $\mathrm{pH} 7.3$ ), 0.1\% sodium dodecylsulfate (SDS), $1 \%$ Triton X-100, $10 \mathrm{mM}$ EDTA, $10 \mathrm{mM}$ sodiumpyrophosphate, $10 \mathrm{mM} \mathrm{NaF}, 125 \mathrm{mM} \mathrm{NaCl}, 1 \%$ protease inhibitor cocktail I, $1 \%$ protease inhibitor cocktail III, and 10\% PhosStop. Cell debris was removed by centrifugation and the lysates were incubated with SDS-sample buffer (final concentrations $50 \mathrm{mM}$ Tris $\mathrm{pH}$ $6.8,4 \%$ glycerin, $0.8 \%$ SDS, $1.6 \% \beta$-mercaptoethanol, and $0.04 \%$ bromophenol blue). Samples were further processed by SDSPAGE and immunoblotting, respectively, as previously described (Dumitru et al., 2012a).

\section{STATISTICAL ANALYSIS}

For the in vitro studies, data are presented as means and SD and statistical analysis was performed with the two-tailed paired Student's $t$-test. Clinical data was analyzed with the SPSS statistical software version 18.0 (SPSS Inc, Chicago, IL, USA). Survival curves (5-years cutoff) were plotted according to the Kaplan-Meier method and significance was tested using univariate analysis (log-rank test) or multivariate Cox regression analysis. Correlation between CORTACTIN phosphorylation and neutrophilic infiltration was tested with Spearmans's rank correlation coefficient (Spearman $\rho$ ). Comparison of: (i) CORTACTIN expression with clinicopathological variables [T-stage and lymph node metastasis (N-stage)] or (ii) CORTACTIN phosphorylation with high versus low neutrophilic infiltration, respectively, was performed with the $\chi^{2}$ test. In all studies, the level of significance was set at $p \leq 0.05$.

\section{RESULTS \\ CORTACTIN IN OROHYPOPHARYNX CARCINOMA: EXPRESSION AND SCORING SYSTEM}

Using AMIDA technology (Rauch et al., 2004) we initially screened for potential tumor-associated antigens in HNC patients (see Materials and Methods). Subsequently, we performed an extensive literature search to select the antigens which might enhance the aggressiveness and progression of tumors. Thus, we identified CORTACTIN as a promising candidate. We then determined the expression of CORTACTIN by immunohistochemical analysis of TMAs from 89 orohypopharynx carcinoma patients. Additionally, we determined CORTACTIN expression in frozen orohypopharynx carcinoma tissues $(n=16)$ versus healthy epithelial tissues from buccal mucosa $(n=12)$. CORTACTIN expression was scored as intensity of staining multiplied by the percentage of positive cells [immunoreactive score (IRS); Figure 1A]. An IRS of 1-4 was considered as weak CORTACTIN, IRS $6-8$ as medium, and IRS 9-12 represented strong CORTACTIN levels (Figure 1A). Interestingly, in healthy epithelial tissues we observed weak and medium levels of CORTACTIN, but no strong CORTACTIN expression (Figure 1B). Consequently, we considered that weak and medium CORTACTIN represent basal levels of the protein (from here 

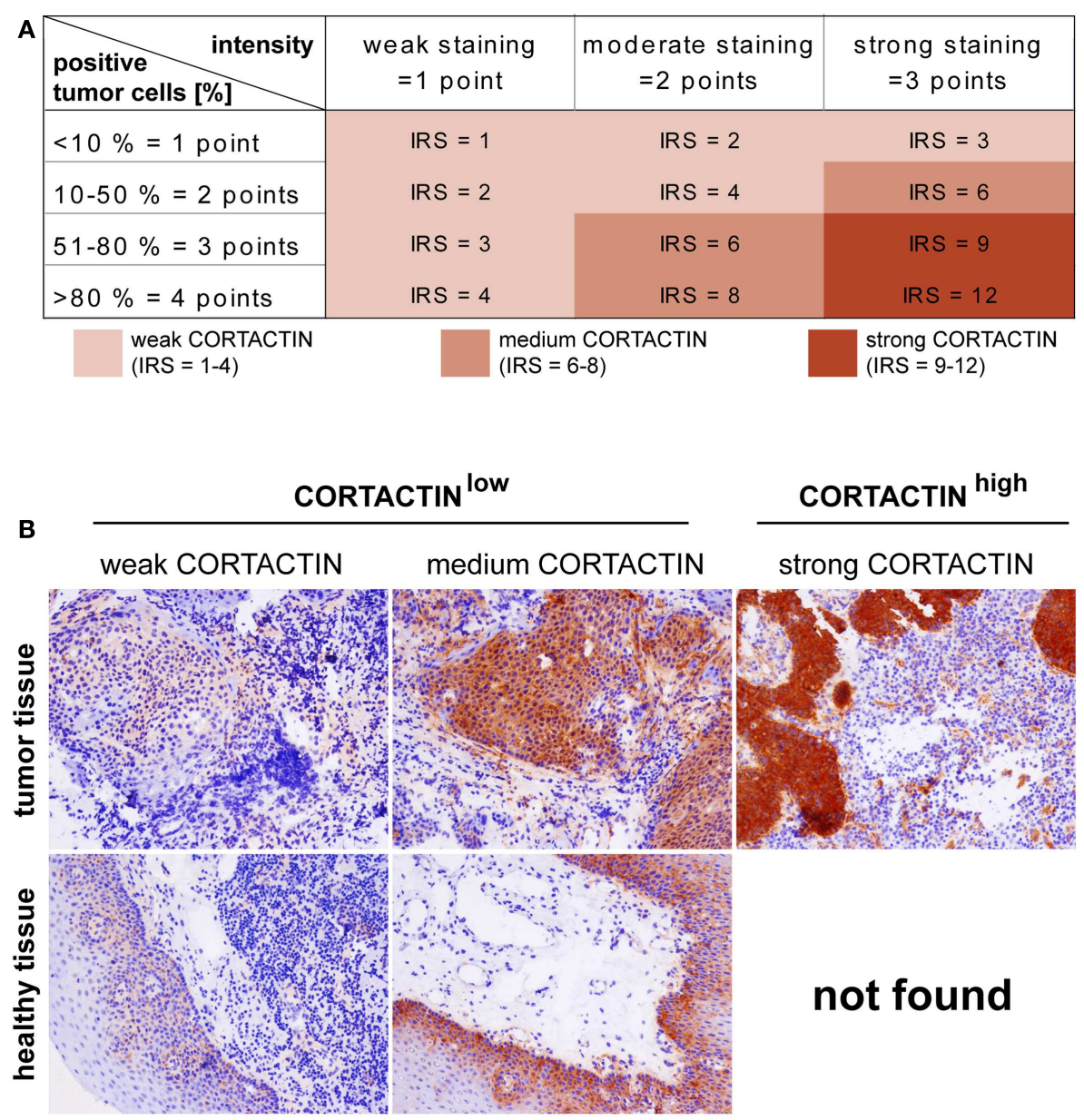

FIGURE 1 | CORTACTIN expression and scoring in tissue microarrays. Malignant orohypopharyngeal and healthy epithelial tissues were stained against CORTACTIN. (A) The immunoreactive score (IRS) was calculated as intensity of the staining reaction multiplied by the percentage of positive cells. Based on the IRS values, CORTACTIN was scored as weak, medium, and strong. (B) Representative micrographs indicating that strong levels of CORTACTIN are found only in malignant tissues, while healthy epithelial tissues displayed either weak or medium levels of CORTACTIN. Subsequently, the weak and medium CORTACTINexpressing specimens were considered as "CORTACTIN" low" while strong CORTACTIN-expressing specimens were termed "CORTACTIN ${ }^{\text {high }}$." Magnification $=200 \times$.

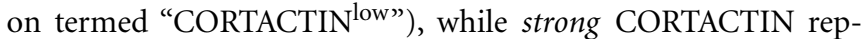
resents overexpressed levels of the protein (from here on termed “CORTACTIN"high").

\section{CORTACTIN AND CLINICAL OUTCOME IN OROHYPOPHARYNX CARCINOMA PATIENTS}

Next, we determined whether CORTACTIN levels associated with 5-years survival, T-stage, and lymph node metastasis (Nstage) in orohypopharynx carcinoma patients. Here and throughout the whole study, T-stage was expressed as T1, T2, T3, T4, while N-stage was expressed as either N0, N1, N2a, N2b, $\mathrm{N} 2 \mathrm{c} / \mathrm{N} 3$ (advanced regional lymphadenopathy), or as unilateral (N1, N2a, N2b) versus bilateral (N2c) lymph node metastasis. The results demonstrated that patients with high levels of tumoral CORTACTIN had significantly shorter overall survival $\left(p=0.028, \log\right.$-rank) than patients with CORTACTIN ${ }^{\text {low }}$ (Figure 2A). Furthermore, $\chi^{2}$ analysis indicated that patients with advanced disease were more likely to express high levels of CORTACTIN than patients with earlier stages of disease $(p=0.027$ for T2 versus T4; $p=0.008$ for T1-2 versus T3-4; $p=0.005$ for $\mathrm{N} 2 \mathrm{a}$ versus $\mathrm{N} 2 \mathrm{c}-3$; $p=0.016$ for $\mathrm{N}$ unilateral versus bilateral). The distribution of patients with high and low expression of CORTACTIN in relation to T-stage and $\mathrm{N}$-stage is depicted in Figures 2B-D.

\section{CORTACTIN AND NEUTROPHILIC INFILTRATION IN OROHYPOPHARYNX CARCINOMA}

Accumulating evidence indicates a critical role of tumorinfiltrating immune cells in the progression of different types of solid cancers. Recently, we demonstrated that high numbers of tumor-infiltrating neutrophils (CD66 $\mathrm{b}^{\text {high }}$ ) associated with higher T-stage and poor survival in orohypopharynx carcinoma patients with advanced disease (Trellakis et al., 2011). Evaluation of the entire cohort of or hypopharynx carcinoma 

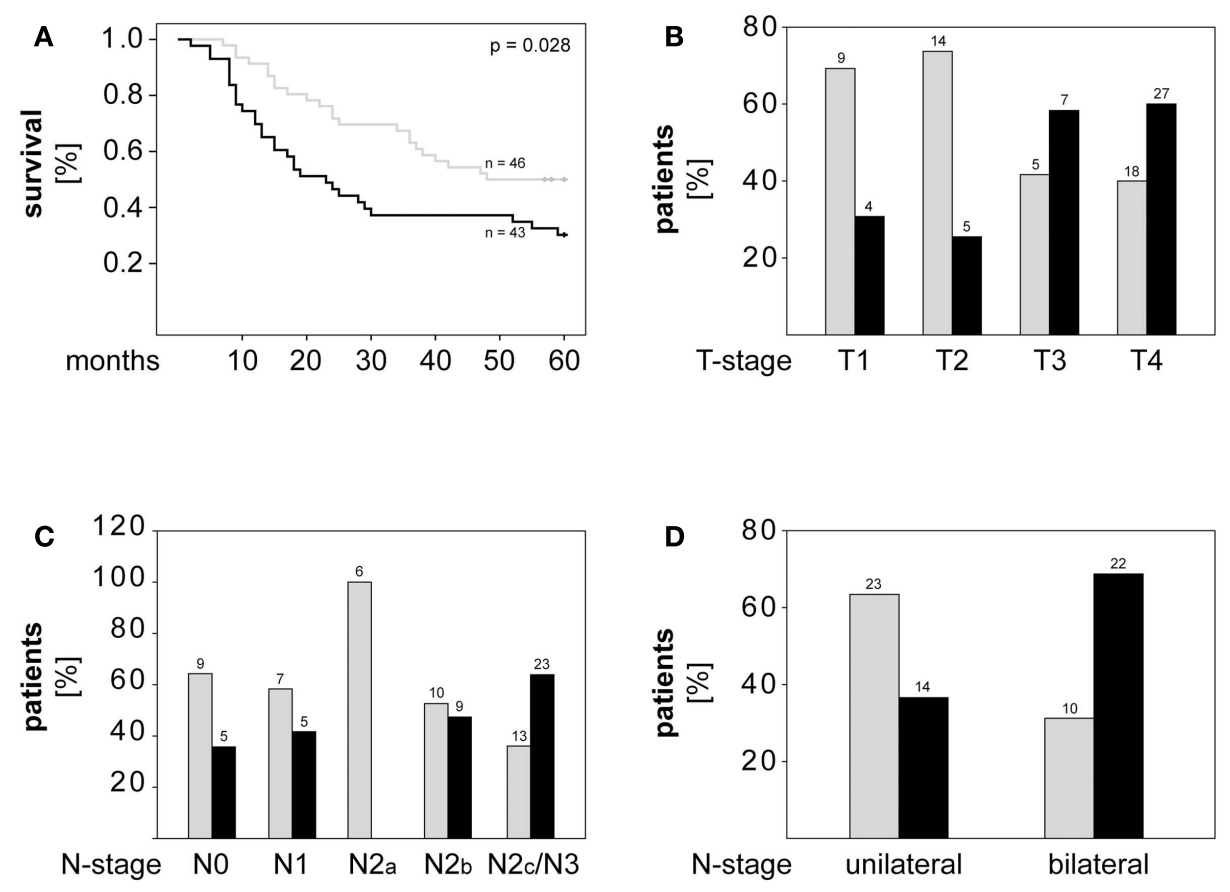

\section{$=$ CORTACTIN ${ }^{\text {IOW }}$}

FIGURE 2 | CORTACTIN overexpression associates with advanced disease and poor clinical outcome in orohypopharynx carcinoma patients. (A) Kaplan-Meier 5-years survival curves were plotted for patients with low versus high tumoral CORTACTIN expression and statistical analysis was performed with the log-rank test. Graphical depiction of (B) T-stage, (C) N-stage, and (D) laterality patients showed that high neutrophilic infiltration tended to associate with poor survival in those patients ( $p=0.054$; Figure 3A). Additionally, we found that patients with advanced $\mathrm{N}$-stage were more likely to exhibit high neutrophilic infiltration than patients with earlier N-stages $(p=0.005$ for $\mathrm{N} 1$ versus $\mathrm{N} 2 \mathrm{c} / \mathrm{N} 3$; $p=0.015$ for $\mathrm{N} 2 \mathrm{~b}$ versus $\mathrm{N} 2 \mathrm{c} / \mathrm{N} 3 ; p=0.001$ for $\mathrm{N}$ unilateral versus bilateral; $\chi^{2}$ ). The distribution of patients with high and low neutrophilic infiltration in relation to $\mathrm{N}$-stage is depicted in Figure 3B.

Next, we investigated the combined effect of CORTACTIN and neutrophilic infiltration on the clinical outcome of orohypopharynx carcinoma patients. To this end, we divided the patients into four groups (CORTACTIN ${ }^{\text {low }} / \mathrm{CD} 6 \mathrm{~b}^{\text {low }}$, CORTACTIN $^{\text {low }} /{\text { CD } 66 b^{\text {high }}}^{\text {, CORTACTIN }}{ }^{\text {high }} / \mathrm{CD} 6 \mathrm{~b}^{\text {low }}$, and CORTACTIN $^{\text {high }} / \mathrm{CD} 6 \mathrm{~b}^{\text {high }}$ ) and determined the survival, Tstage, and the rate of lymph node metastasis. The results demonstrated that CORTACTIN ${ }^{\text {high }} /$ CD66 $b^{\text {high }}$ patients had the shortest overall survival ( $p=0.010$, log-rank; Figure 4A). In further analysis we performed $\chi^{2}$ tests for CORTACTIN ${ }^{\text {high }} /{\text { CD } 66 b^{\text {high }}}^{\text {patients }}$ versus the other three groups of patients taken together. The results indicated that patients with advanced T-stage were more likely to express synchronous high levels of CORTACTIN and neutrophilic infiltration than patients with earlier stages of disease $(p=0.036$ for $\mathrm{T} 1$ versus $\mathrm{T} 4 ; \mathrm{p}=0.041$ for $\mathrm{T} 2$ versus $\mathrm{T} 3 ; p<0.001$ for $\mathrm{T} 2$ versus T4; $p<0.001$ for T1-2 versus T3-4). Similar results were obtained for patients with advanced N-stage $(p=0.042$ for N0 versus $\mathrm{N} 2 \mathrm{c} / \mathrm{N} 3 ; p=0.007$ for $\mathrm{N} 1$ versus $\mathrm{N} 2 \mathrm{c} / \mathrm{N} 3 ; p=0.023$ for $\mathrm{N} 2 \mathrm{a}$ versus $\mathrm{N} 2 \mathrm{c} / \mathrm{N} 3 ; p=0.003$ for $\mathrm{N} 2 \mathrm{~b}$ versus $\mathrm{N} 2 \mathrm{c} / \mathrm{N} 3 ; p<0.001$ for $\mathrm{N}$ unilateral versus bilateral). The distribution of patients with various combinations of CORTACTIN/CD66b levels in relation to T-stage and N-stage is depicted in Figures 4B-D. To confirm the prognostic value of CORTACTIN and neutrophilic infiltration for the survival of orohypopharynx carcinoma patients, we performed multivariate Cox regression analysis in a model adjusted for the type of therapy received, smoking/alcohol consumption and gender. The results showed that the CORTACTIN ${ }^{\text {high }} /{\text { CD } 66 b^{\text {high }}}^{\text {phe- }}$ notype was the only significant predictor for poor survival in these patients $(\mathrm{HR}=2.34,95 \% \mathrm{CI}=1.01-5.38, p=0.045$; Table 2$)$. Taken together these data indicate that only the simultaneous presence of high tumoral CORTACTIN levels and high numbers of neutrophils associates with poor clinical outcome in orohypopharynx carcinoma patients. Furthermore, our findings suggest that CORTACTIN and neutrophils might functionally "cooperate" to enhance the progression of orohypopharynx carcinoma in vivo. 

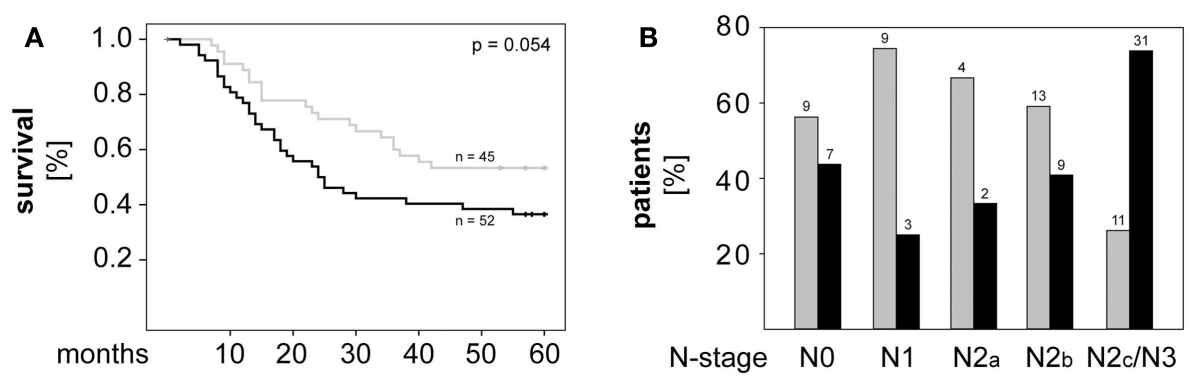

$=\mathrm{CD} 6 \mathrm{~b}^{\text {low }}$

(CD66b low numbers)

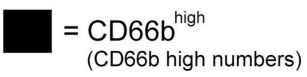

FIGURE 3 | High neutrophilic infiltration associates with poor survival and metastasis to the lymph nodes in orohypopharynx carcinoma patients. (A) Kaplan-Meier 5-years survival curves were plotted for patients with low versus high neutrophilic infiltration and statistical analysis was performed with the log-rank test. (B) Graphical depiction of N-stage in patients with high versus low neutrophilic infiltration. Statistical testing was performed with the $\chi^{2}$ test $(p=0.005$ for $\mathrm{N} 1$ versus N2c/N3; $p=0.015$ for $\mathrm{N} 2 \mathrm{~b}$ versus N2c/N3; $p=0.001$ for $\mathrm{N}$ unilateral versus bilateral). Patients with high numbers of infiltrating neutrophils (CD66 $\mathrm{b}^{\text {high }}$ ) are depicted in black, while patients with low numbers of infiltrating neutrophils ( $\left.C D 66 b^{\text {low }}\right)$ are depicted in gray. The absolute patient numbers are indicated above each bar of the graphs.
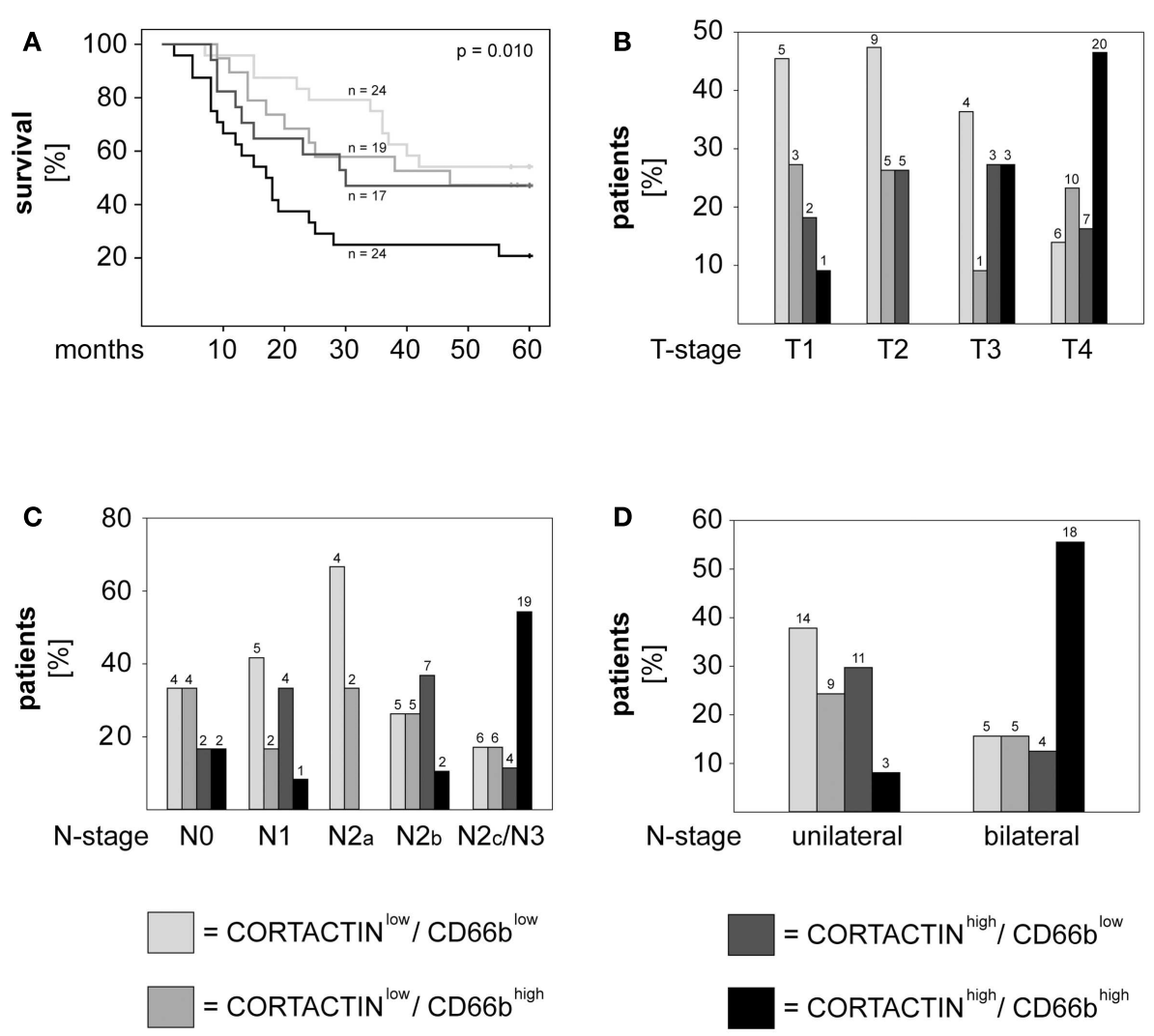

FIGURE 4 | High CORTACTIN and high neutrophilic infiltration (CD66b) associate with the most unfavorable clinical outcome. Analysis of (A) 5-years survival, (B) T-stage, (C) N-stage, and (D) laterality of lymph node metastasis in patients with various combinations of CORTACTIN levels and neutrophilic infiltration, respectively. Statistical testing was performed with the log-rank test (for survival) and with the $\chi^{2}$

test for the remaining clinical parameters $(p=0.036$ for T1 versus T4; $p=0.041$ for T2 versus T3; $p<0.001$ for T2 versus T4; $p<0.001$ for T1 2 versus T3-4; $p=0.042$ for $\mathrm{N} 0$ versus N2c/N3; $p=0.007$ for $\mathrm{N} 1$ versus $\mathrm{N} 2 \mathrm{c} / \mathrm{N} 3 ; p=0.023$ for $\mathrm{N} 2 \mathrm{a}$ versus $\mathrm{N} 2 \mathrm{c} / \mathrm{N} 3 ; p=0.003$ for $\mathrm{N} 2 \mathrm{~b}$ versus $\mathrm{N} 2 \mathrm{c} / \mathrm{N} 3 ; p<0.001$ for $\mathrm{N}$ unilateral versus bilateral). The absolute patient numbers are indicated above each bar of the graphs. 
Table 2 | Multivariate Cox regression analysis of CORTACTIN and neutrophilic infiltration adjusted for type of therapy, smoking/alcohol consumption, and gender.

\begin{tabular}{|c|c|c|c|}
\hline \multirow[t]{2}{*}{ Cox regression multivariate } & \multicolumn{3}{|c|}{ Hazard ratio for survival } \\
\hline & HR & $95 \% \mathrm{Cl}$ & $p$-Value \\
\hline \multicolumn{4}{|l|}{ CORTACTIN/CD66b } \\
\hline CORTACTIN ${ }^{\text {low }} /$ CD66b low & 1 & & \\
\hline CORTACTIN low/CD66 $\mathrm{b}^{\text {high }}$ & 1.26 & $0.50-3.19$ & 0.617 \\
\hline CORTACTIN ${ }^{\text {high }} / C D 66 b^{\text {low }}$ & 1.37 & $0.54-3.50$ & 0.502 \\
\hline CORTACTIN high/CD66 $b^{\text {high }}$ & 2.34 & $1.01-5.38$ & $0.045^{*}$ \\
\hline \multicolumn{4}{|l|}{ THERAPY } \\
\hline Surgery & 1 & & \\
\hline Radio(chemo)therapy & 1.61 & $0.41-6.36$ & 0.493 \\
\hline Surgery + radio(chemo)therapy & 1.34 & $0.36-4.98$ & 0.662 \\
\hline \multicolumn{4}{|l|}{ ALCOHOL/TOBACCO } \\
\hline None & 1 & & \\
\hline Tobacco & 0.97 & $0.28-3.37$ & 0.970 \\
\hline Alcohol & 0.61 & $0.06-6.12$ & 0.611 \\
\hline Both & 1.39 & $0.57-3.79$ & 0.518 \\
\hline \multicolumn{4}{|l|}{ GENDER } \\
\hline Female & 1 & & \\
\hline Male & 2.04 & $0.88-4.73$ & 0.093 \\
\hline
\end{tabular}

$H R$, hazard ratio; $95 \% \mathrm{Cl}, 95 \%$ confidence interval. The asterisk (*) indicates the parameters showing statistical significance $(p \leq 0.05)$.

\section{TUMORAL CORTACTIN AND NEUTROPHILS: MECHANISMS OF INTERACTION}

In a further set of studies we sought to clarify the relationship between tumoral CORTACTIN and neutrophils by using an in vitro system well established in our group and previously described (Dumitru et al., 2011). This system mimics the reciprocal (bi-directional) interaction of tumor cells and neutrophils in the tumor microenvironment. As illustrated in Figure 5A, neutrophils are "primed" by tumoral factors and, subsequently, exert feed-back effects on the tumor cells (Figure 5A). We initially tested whether neutrophils might upregulate the expression of CORTACTIN in the tumor cells. To this end, FaDu cells (hypopharynx carcinoma) were stimulated with factors released by the "primed" neutrophils ( $\mathrm{FaDu}$ /neutrophil $\mathrm{SN}$ ). As control we used supernatants containing only tumoral factors (FaDu $\mathrm{SN}$ ) or supernatants from "un-primed" neutrophils (neutrophil $\mathrm{SN}$ ). We initially assessed the total levels of CORTACTIN in a time-course by western blot. We did not observe a significant change in CORTACTIN expression upon stimulation by neutrophils (Figure 5B - upper panel). However, when the samples were tested for phosphorylated CORTACTIN, we found increased levels of phospho-CORTACTIN in tumor cells exposed to factors released by the "primed" neutrophils (Figure 5B - middle panel).

Next, we tested whether neutrophil-induced phosphorylation of CORTACTIN might occur also in vivo. To this end, we stained consecutive sections from frozen orohypopharynx carcinoma tissues (37 patients) against total and phosphorylated (Tyr421) forms of CORTACTIN, respectively. All samples were co-stained against CD66b (neutrophil marker). Micrographs were taken at 100 -fold magnification from the same region of the tumor tissue for CORTACTIN or phospho-CORTACTIN (each with CD66b co-staining). The micrographs were analyzed with the ImageJ software and the values obtained were considered as Arbitrary Fluorescent Units (AFU). We then plotted the levels of CORTACTIN phosphorylation [phospho-CORTACTIN (AFU)] against the levels of neutrophilic infiltration [CD66b (AFU); Figure 6A] . Statistical analysis using Spearman's rank test indicated that CORTACTIN phosphorylation significantly correlated with neutrophilic infiltration $(p<0.001, \rho=0.620)$. Additionally, we divided the patients into $\mathrm{CD} 6 \mathrm{~b}^{\text {low }}$ and $\mathrm{CD} 66 \mathrm{~b}^{\text {high }}$ according to the median value of CD66b (AFU) and found that CD66 $\mathrm{b}^{\text {high }}$ patients had significantly higher CORTACTIN phosphorylation than $\mathrm{CD}_{6} 6 \mathrm{~b}^{\text {low }}$ patients $\left(p=0.029, \chi^{2}\right.$; Figure 6B). A representative example of CORTACTIN phosphorylation in CD66b ${ }^{\text {low }}$ versus CD66b ${ }^{\text {high }}$ patients is shown in Figure 6C. Taken together, our in vitro and in situ studies demonstrate that neutrophils interact with the tumor cells by inducing CORTACTIN phosphorylation and activation.

\section{TUMORAL CORTACTIN - NEUTROPHIL INTERACTION: FUNCTIONAL CONSEOUENCES}

Finally, we investigated which functional changes might neutrophils induce in the tumor cells via CORTACTIN. Based on the findings that: (i) CORTACTIN is an important regulator of cellular migration (Ammer and Weed, 2008) and (ii) neutrophils enhance the migration of HNC cells (Dumitru et al., 2011), we hypothesized that neutrophils "exploit" high tumoral CORTACTIN levels to promote the migration of the tumor cells. Conversely, in the absence of tumoral CORTACTIN, neutrophils would not be able to stimulate the migration of the tumor cells. We, therefore, knocked-down CORTACTIN in FaDu cells by siRNA. As control, $\mathrm{FaDu}$ cells were transfected with mock siRNA (Figures 7A,B). We then determined the migratory potential of the tumor cells in the presence or absence of factors released by "primed" neutrophils. The results showed that mock-transfected FaDu cells displayed accelerated migration in response to neutrophil stimulation, as indicated by enhanced closure of the detection zone ("gap"; Figure 7C). In contrast, this effect of neutrophil stimulation was abolished in CORTACTIN knock-down FaDu cells (Figure 7C). These findings indicate that, consistent with the results presented in Figure 4, high levels of tumoral CORTACTIN are required for neutrophil-induced tumoral migration and progression.

\section{DISCUSSION}

Increased effort has been made to identify cellular/molecular factors that could provide accurate information regarding cancer diagnosis, prognosis, and response to therapy. In this study, we identify tumoral CORTACTIN as a prognostic factor for the clinical outcome in orohypopharynx carcinoma patients. More importantly, we demonstrate that combined analysis of tumoral CORTACTIN and of neutrophilic infiltration in the tumor tissue has higher prognostic impact for the clinical outcome in these patients. In addition to the clinical findings, our study provides a biological and mechanistical link between neutrophils and 

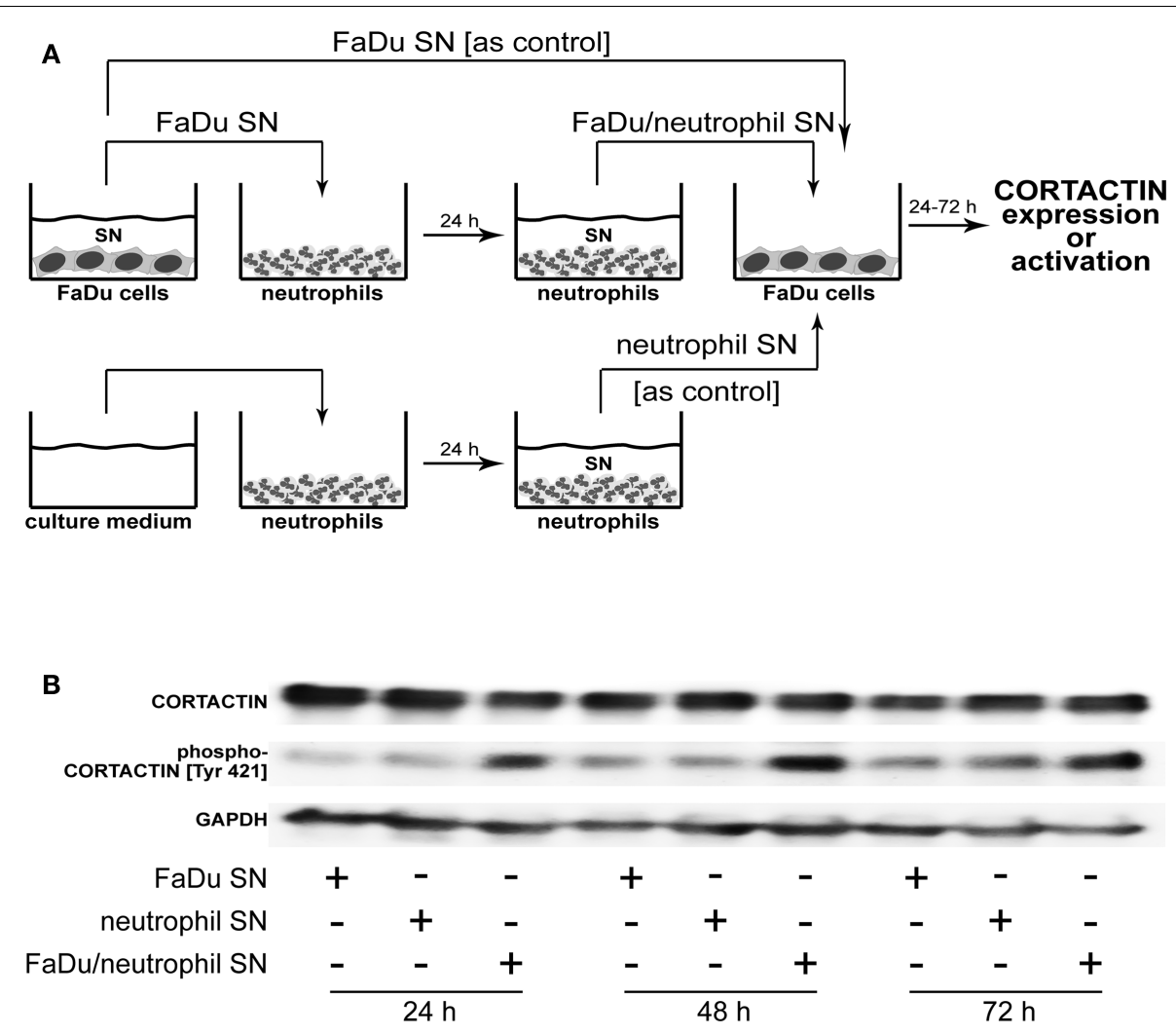

FIGURE 5 | Neutrophils phosphorylate CORTACTIN in FaDu (hypopharynx carcinoma) cells. (A) Neutrophils were "primed" by co-incubation with FaDu supernatants (FaDu SN) for $24 \mathrm{~h}$. The resulting supernatants (FaDu/neutrophil SN) were used to stimulate FaDu cells for 24, 48 , or 72 h. FaDu SN or neutrophil SN only were used as controls. (B)
CORTACTIN expression and activation were assessed by western blot using antibodies against total CORTACTIN (upper panel) or phosphorylated CORTACTIN (Tyr 421; middle panel). GAPDH (lower panel) was used as control for the quantity and quality of protein lysates. The results are representative for five independent experiments each. tumoral CORTACTIN by showing that neutrophils phosphorylate CORTACTIN in the tumor cells to promote their migration.

The potential role of CORTACTIN as prognostic biomarker has been previously investigated in different types of cancer (Li et al., 2008; Wang et al., 2009; Cai et al., 2010; Xu et al., 2010). Several studies observed that CORTACTIN associated with poor clinical outcome also in HNC patients (Gibcus et al., 2008; Hofman et al., 2008; Rodrigo et al., 2009). Among the HNC subtypes, CORTACTIN has been mainly proposed as a potential biomarker for laryngeal carcinoma (Gibcus et al., 2008; Rodrigo et al., 2009). Surprisingly, the study of Rodrigo et al. (2009) suggested that, while having prognostic value in laryngeal carcinoma, CORTACTIN does not associate with the clinical outcome in pharyngeal carcinoma, which is in contrast to our findings. This discrepancy might result from the different read-outs used to assess CORTACTIN (over)expression. While we analyzed directly the protein levels of CORTACTIN in relation to clinical parameters, the abovementioned studies based their statistical analysis mainly on the gene amplification of CORTACTIN. However, it has been often shown that protein and gene expression do not perfectly correlate. In fact, even the study of Rodrigo et al. (2009) found that gene amplification of CORTACTIN did not correlate to the protein levels observed by immunohistochemistry in 35\% of specimens.
Yet proteins are the ultimate effectors in biological processes and analysis of protein levels is likely to provide more reliable biological insights than analysis of gene expression. Thus, by quantifying the protein expression of CORTACTIN, our study sheds a new light on the role of this molecule in the prognosis of HNC.

Of novelty and significance are our findings regarding the interaction between tumoral CORTACTIN and neutrophils (CD66bpositive cells) in orohypopharynx carcinoma. As outlined above, an association of either CORTACTIN or tumor-infiltrating neutrophils with poor clinical outcome in cancer patients has already been proposed by previous studies (Donskov and von der Maase, 2006; Jensen et al., 2009, 2012; Kuang et al., 2011; Trellakis et al., 2011; Ilie et al., 2012). However, a link between CORTACTIN and neutrophils has not been shown in any type of cancer thus far. Here, we demonstrate that patients exhibiting high levels of tumoral CORTACTIN together with high neutrophilic infiltra-

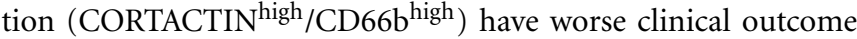
than all other groups of patients in our cohort. These findings are further substantiated by multivariate analysis adjusted for factors potentially relevant for progression of orohypopharynx carcinoma, such as smoking/alcohol consumption, type of therapy, and gender. Within this model, CORTACTIN ${ }^{\text {high }} /$ CD $66 b^{\text {high }}$ phenotype was the only significant predictor of poor survival for 

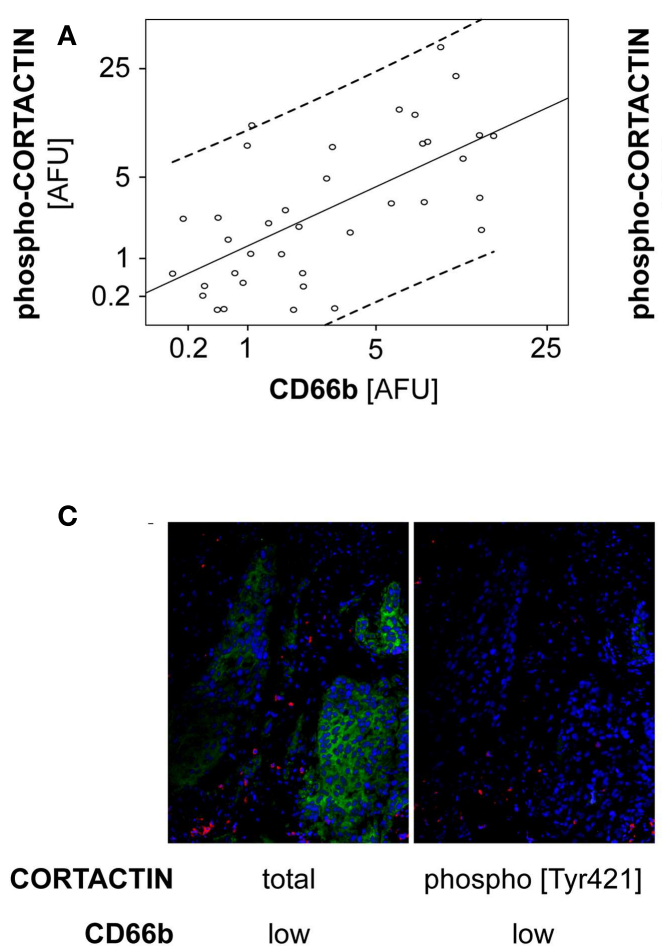

phospho [Tyr421]

low
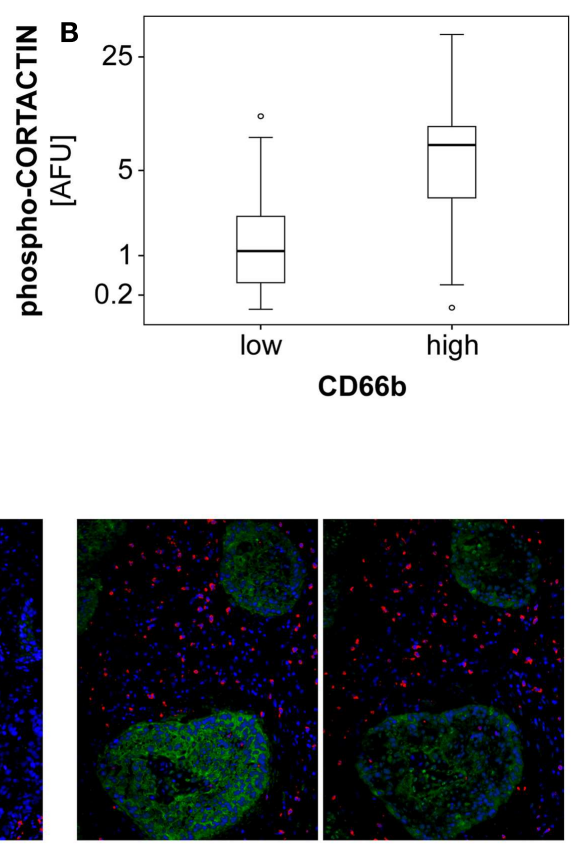

total

high

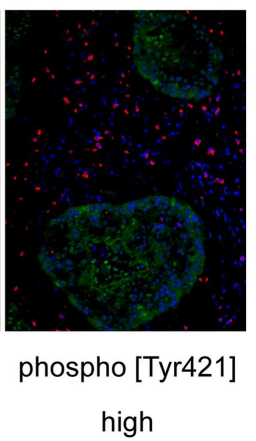

high
FIGURE 6 | Phosphorylated CORTACTIN associates with neutrophilic infiltration in orohypopharynx carcinoma tissues. Consecutive tumor tissue sections from 37 orohypopharynx carcinoma patients were stained against CORTACTIN/CD66b or phospho-CORTACTIN/CD66b. Total CORTACTIN, phospho-CORTACTIN, and neutrophilic infiltration were analyzed with the ImageJ software. (A) Correlation between neutrophilic infiltration and the levels of CORTACTIN phosphorylation. Dotted lines indicate the $95 \% \mathrm{Cl}$ (confidence interval) for the regression line. Statistical analysis was performed with Spearman's rank correlation coefficient $(p<0.001 ; \rho=0.620)$. (B) The level of CORTACTIN phosphorylation in patients with low $(n=19)$ versus high $(n=18)$ neutrophilic infiltration. Shown are the medians (black lines) and percentiles (25th and 75th) as vertical boxes with error bars; outliers are indicated as small circles. Statistical analysis was performed with the $\chi^{2}$ test $(p=0.029)$. (C) Representative micrographs (magnification $=200 \times$ ) of CORTACTIN phosphorylation in patients with low versus high neutrophilic infiltration. Green, CORTACTIN or phospho-CORTACTIN; red, CD66b (neutrophils); blue pseudocolor, DRAQ5 (nuclear staining). orohypopharynx carcinoma patients. Although necessary to be confirmed by a larger study incorporating all REMARK guidelines, CORTACTIN, and tumor-infiltrating neutrophils seem, nevertheless, to be promising biomarker candidates for an accurate prognosis in orohypopharynx carcinoma.

Our clinical findings also suggest that neutrophils (indirectly) interact with the tumor cells via CORTACTIN to enhance the progression of orohypopharynx carcinoma. This interaction is confirmed by our in vitro and in situ studies which show that neutrophils activate (phosphorylate) tumoral CORTACTIN to promote the migration of the tumor cells. These findings are highly relevant, since the molecular mechanisms of tumor-neutrophil interactions are still not completely understood. Previous studies showed that neutrophils have pro-angiogenic effects, or promote migration, invasion, and proliferation of the tumor cells (Gregory and Houghton, 2011; Dumitru et al., 2012b). With regard to regulation of tumoral motility/migration by neutrophils, several potential mechanisms have been recently proposed. In breast carcinoma, neutrophils were shown to cluster tumoral ICAM1 and phosphorylate FAK (focal adhesion kinase) and paxillin via src, as well as p38-MAPK via Rho-GTPase (Strell et al., 2010). In hepatocellular carcinoma, neutrophils were activated by tumor-derived hyaluronan binding to TLR4 on their surface and promoted tumor cell motility (Wu et al., 2011). Notably, both of the above-mentioned studies indicated that the pro-migratory effects of neutrophils are mediated via direct cell-to-cell contact. In our system, neutrophils activated CORTACTIN and enhanced tumor migration via release of soluble factors. Thus, besides supporting the association of CORTACTIN and neutrophils with increased metastasis in cancer patients, these findings unravel a novel mechanism of neutrophil-mediated tumor migration and progression. We certainly do not exclude that, other infiltrating immune cells (in particular macrophages) enhance the aggressiveness of orohypopharynx carcinoma in vivo. However, extensive work is still required to dissect the relative importance of various immune cell subsets in progression of solid cancers.

In summary, our study combines clinical and experimental approaches to provide evidence for a clinically relevant mechanism of tumor immunomodulation by stromal neutrophils. Ultimately, these findings contribute to a better understanding of the tumor microenvironment and foster the development of improved therapeutic strategies against orohypopharynx carcinoma and, perhaps, other types of solid cancer as well. 

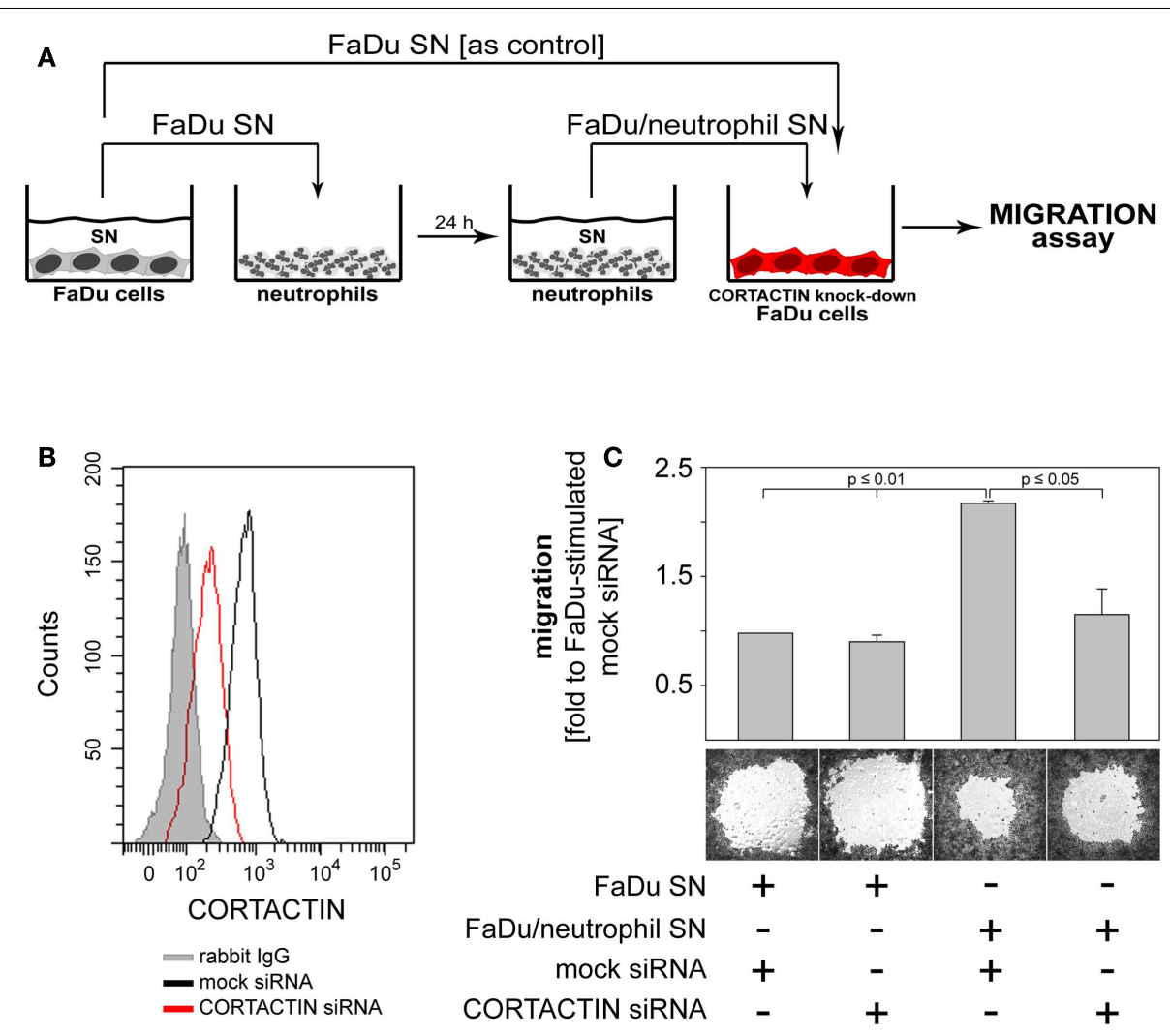

FIGURE 7 | Neutrophils promote the migration of FaDu cells via

CORTACTIN. (A) FaDu cells in which CORTACTIN had been knocked-down by siRNA were stimulated with FaDu/neutrophil SN and migration was assessed $48 \mathrm{~h}$ later. (B) Efficiency of CORTACTIN knock-down was determined by flow cytometric analysis of total CORTACTIN levels at $72 \mathrm{~h}$ post-transfection. (C) FaDu cells (transfected with CORTACTIN or mock siRNA) were grown to confluency in the presence of "gap"-forming culture inserts. The "gap" refers to the cell-free round area visible in the figure. Stimulation was performed as indicated in A. Cells were fixed/stained with a solution containing crystal violet and micrographs were taken at 25-fold magnification. "Gap"-closure was quantified with the ImageJ software. Data are means \pm SD of three independent experiments (upper panel). A representative result is shown in the lower panel of the figure. Statistical testing was performed with the two-tailed paired Student's $t$-test.

\section{ACKNOWLEDGMENTS}

We thank Petra Altenhoff and Sebastian Vollmer (Department of Otorhynolaryngology, University of Duisburg-Essen) and Anja Peglow (Department of Pathology/Neuropathology,

\section{REFERENCES}

Ammer, A. G., and Weed, S. A. (2008). Cortactin branches out: roles in regulating protrusive actin dynamics. Cell Motil. Cytoskeleton 65, 687-707.

Bekes, E. M., Schweighofer, B., Kupriyanova, T. A., Zajac, E., Ardi, V. C., Quigley, J. P., et al. (2011). Tumor-recruited neutrophils and neutrophil TIMP-free MMP-9 regulate coordinately the levels of tumor angiogenesis and efficiency of malignant cell intravasation. Am. J. Pathol. 179, 1455-1470.

Cai, J. H., Zhao, R., Zhu, J. W., Jin, X. L., Wan, F. J., Liu, K., et al. (2010). Expression of cortactin correlates with a poor prognosis in patients with stages II-III colorectal adenocarcinoma. J. Gastrointest. Surg. 14, 1248-1257.

Choong, N., and Vokes, E. (2008). Expanding role of the medical oncologist in the management of head and neck cancer. CA Cancer J. Clin. 58, 32-53.

Coussens, L. M., and Werb, Z. (2002). Inflammation and cancer. Nature 420, 860-867.

Donskov, F., and von der Maase, H. (2006). Impact of immune parameters on long-term survival in metastatic renal cell carcinoma. $J$. Clin. Oncol. 24, 1997-2005.

Dumitru, C. A., Fechner, M. K., Hoffmann, T. K., Lang, S., and Brandau, S. (2012a). A novel p38-MAPK

University of Duisburg-Essen) for excellent technical support. This study was supported in part by the German Ministry for Education and Research (BMBF) and by Krebsgesellschaft NRW.

signaling axis modulates neutrophil biology in head and neck cancer. $J$. Leukoc. Biol. 91, 591-598.

Dumitru, C. A., Moses, K., Trellakis, S., Lang, S., and Brandau, S. (2012b). Neutrophils and granulocytic myeloid-derived suppressor cells: immunophenotyping, cell biology and clinical relevance in human oncology. Cancer Immunol. Immunother. 61, 1155-1167.

Dumitru, C. A., Gholaman, H., Trellakis, S., Bruderek, K., Dominas, N., $\mathrm{Gu}, \mathrm{X}$., et al. (2011). Tumor-derived macrophage migration inhibitory factor modulates the biology of head and neck cancer cells via neutrophil activation. Int. J. Cancer 129, 859-869.
Gibcus, J. H., Mastik, M. F., Menkema, L., de Bock, G. H., Kluin, P. M., Schuuring, E., et al. (2008). Cortactin expression predicts poor survival in laryngeal carcinoma. Br. J. Cancer 98, 950-955.

Gregory, A. D., and Houghton, A. M. (2011). Tumor-associated neutrophils: new targets for cancer therapy. Cancer Res. 71, 2411-2416.

Hofman, P., Butori, C., Havet, K., Hofman, V., Selva, E., Guevara, N., et al. (2008). Prognostic significance of cortactin levels in head and neck squamous cell carcinoma: comparison with epidermal growth factor receptor status. Br. J. Cancer 98, 956-964. 
Ilie, M., Hofman, V., Ortholan, C., Bonnetaud, C., Coelle, C., Mouroux, J., et al. (2012). Predictive clinical outcome of the intratumoral CD66b-positive neutrophil-toCD8-positive T-cell ratio in patients with resectable nonsmall cell lung cancer. Cancer 118, 1726-1737.

Jablonska, J., Leschner, S., Westphal, K., Lienenklaus, S., and Weiss, S. (2010). Neutrophils responsive to endogenous IFN-beta regulate tumor angiogenesis and growth in a mouse tumor model. J. Clin. Invest. 120, 1151-1164.

Jemal, A., Siegel, R., Ward, E., Murray, T., $\mathrm{Xu}$, J., and Thun, M. J. (2007). Cancer statistics, 2007. CA Cancer J. Clin. 57, 43-66.

Jensen, H. K., Donskov, F., Marcussen, N., Nordsmark, M., Lundbeck, F., and von der Maase, H. (2009). Presence of intratumoral neutrophils is an independent prognostic factor in localized renal cell carcinoma. $J$. Clin. Oncol. 27, 4709-4717.

Jensen, T. O., Schmidt, H., Moller, H. J., Donskov, F., Hoyer, M., Sjoegren, P., et al. (2012). Intratumoral neutrophils and plasmacytoid dendritic cells indicate poor prognosis and are associated with pSTAT3 expression in AJCC stage I/II melanoma. Cancer 118, 2476-2485.
Kuang, D. M., Zhao, Q., Wu, Y., Peng, C., Wang, J., Xu, Z., et al. (2011). Peritumoral neutrophils link inflammatory response to disease progression by fostering angiogenesis in hepatocellular carcinoma. J. Hepatol. 54, 948-955.

Li, X., Zheng, H., Hara, T., Takahashi, H., Masuda, S., Wang, Z., et al. (2008). Aberrant expression of cortactin and fascin are effective markers for pathogenesis, invasion, metastasis and prognosis of gastric carcinomas. Int. J. Oncol. 33, 69-79.

Lin, W. W., and Karin, M. (2007) A cytokine-mediated link between innate immunity, inflammation, and cancer. J. Clin. Invest. 117, 1175-1183.

Nozawa, H., Chiu, C., and Hanahan, D. (2006). Infiltrating neutrophils mediate the initial angiogenic switch in a mouse model of multistage carcinogenesis. Proc. Natl. Acad. Sci. U.S.A. 103, 12493-12498.

Rauch, J., Ahlemann, M., Schaffrik, M., Mack, B., Ertongur, S., Andratschke, M., et al. (2004). Allogenic antibodymediated identification of head and neck cancer antigens. Biochem. Biophys. Res. Commun. 323, 156-162.

Rodrigo, J. P., Garcia-Carracedo, D., Garcia, L. A., Menendez, S., Allonca, E., Gonzalez, M. V., et al. (2009). Distinctive clinicopathological associations of amplification of the cortactin gene at $11 \mathrm{q} 13$ in head and neck squamous cell carcinomas. $J$. Pathol. 217, 516-523.

Strell, C., Lang, K., Niggemann, B. Zaenker, K. S., and Entschladen, F. (2010). Neutrophil granulocytes promote the migratory activity of MDA-MB-468 human breast carcinoma cells via ICAM-1. Exp. Cell Res. 316, 138-148.

Trellakis, S., Bruderek, K., Dumitru, C. A., Gholaman, H., Gu, X., Bankfalvi, A., et al. (2011). Polymorphonuclear granulocytes in human head and neck cancer: Enhanced inflammatory activity, modulation by cancer cells and expansion in advanced disease. Int. J. Cancer 129, 2183-2193.

Wang, G. C., Hsieh, P. S., Hsu, H. H., Sun, G. H., Nieh, S., Yu, C. P., et al. (2009). Expression of cortactin and survivin in renal cell carcinoma associated with tumor aggressiveness. World J. Urol. 27, 557-563.

Wu, Y., Zhao, Q., Peng, C., Sun, L., Li, X. F., and Kuang, D. M. (2011). Neutrophils promote motility of cancer cells via a hyaluronan-mediated TLR4/PI3K activation loop. J. Pathol. 225, 438-447.

Xu, X. Z., Garcia, M. V., Li, T. Y., Khor, L. Y., Gajapathy, R. S., Spittle, C., et al. (2010). Cytoskeleton alterations in melanoma: aberrant expression of cortactin, an actin-binding adapter protein, correlates with melanocytic tumor progression. Mod. Pathol. 23, 187-196.

Conflict of Interest Statement: The authors declare that the research was conducted in the absence of any commercial or financial relationships that could be construed as a potential conflict of interest.

Received: 23 November 2012; accepted: 30 January 2013; published online: 18 February 2013.

Citation: Dumitru CA, Bankfalvi A, Gu X, Eberhardt WE, Zeidler $R$, Lang $S$ and Brandau S (2013) Neutrophils activate tumoral CORTACTIN to enhance progression of orohypopharynx carcinoma. Front. Immun. 4:33. doi: 10.3389/fimmu.2013.00033

This article was submitted to Frontiers in Tumor Immunity, a specialty of Frontiers in Immunology.

Copyright (C) 2013 Dumitru, Bankfalvi, Gu, Eberhardt, Zeidler, Lang and Brandau. This is an open-access article distributed under the terms of the Creative Commons Attribution License, which permits use, distribution and reproduction in other forums, provided the original authors and source are credited and subject to any copyright notices concerning any third-party graphics etc. 